\title{
Latest results from the Pamela experiment
}

\author{
Oscar Adriani on behalf of the Pamela Collaboration* \\ University of Florence and INFN Sezione di Firenze, Italy \\ E-mail: adriani@fi.infn.it
}

O. Adriani ${ }^{a, b}$, G. C. Barbarino ${ }^{c, d}$, G. A. Bazilevskaya ${ }^{e}$, R. Bellotti ${ }^{f, g}$, M. Boezio $^{h}$, E. A.

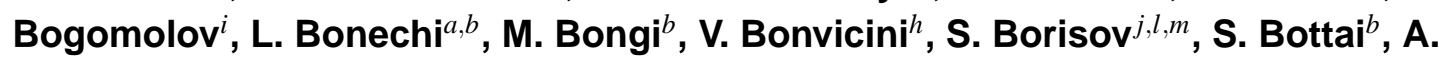
Bruno $^{f, g}$, F. Cafagna ${ }^{g}$, D. Campana ${ }^{d}$, R. Carbone ${ }^{j, d}$, P. Carlson ${ }^{k}$, M. Casolino $^{l}$, G. Castellini $^{n}$, L. Consiglio ${ }^{d}$, M. P. De Pascale ${ }^{j, l}$, C. De Santis ${ }^{l}$, N. De Simone ${ }^{j, l}$, V. Di Felice $^{j, l}$, A. M. Galper ${ }^{m}$, L. Grishantseva ${ }^{m}$, W. Gillard ${ }^{l}$, P. Hofverberg ${ }^{l}$, G. Jerse ${ }^{h, o}$, S. V. Koldashov ${ }^{m}$, S. Y. Krutkov ${ }^{i}$, A. N. Kvashnin ${ }^{e}$, A. Leonov ${ }^{m}$, V. Malvezzi ${ }^{l}$, L. Marcelli $^{l}$, W. Menn ${ }^{p}$, V. V. Mikhailov ${ }^{m}$, E. Mocchiutti ${ }^{h}$, N. Mori ${ }^{b}$, N. Nikonov ${ }^{j, l, i}$, G. Osteria $^{d}$, P. Papini ${ }^{b}$, M. Pearce ${ }^{k}$, P. Picozza ${ }^{j, l}$, M. Ricci $^{q}$, S. B. Ricciarini ${ }^{b}$, M. Simon $^{p}$, R. Sparvoli ${ }^{j}, l$, P. Spillantini ${ }^{a, b}$, Y. I. Stozhkov ${ }^{e}$, A. Vacchi $^{h}$, E. Vannuccini ${ }^{b}$, G. Vasilyev $^{g}$, S. A. Voronov ${ }^{m}$, Y. T. Yurkin ${ }^{m}$, G. Zampa ${ }^{h}$, N. Zampa ${ }^{h}$ and V. G. Zverev ${ }^{m}$

${ }^{a}$ University of Florence, Department of Physics, Via Sansone 1, I-50019 Sesto Fiorentino,

Florence, Italy.

${ }^{b}$ INFN, Sezione di Florence, Via Sansone 1, I-50019 Sesto Fiorentino, Florence, Italy.

'University of Naples "Federico II', Department of Physics, Via Cintia, I-80126 Naples, Italy.

'INFN, Sezione di Naples, Via Cintia, I-80126 Naples, Italy.

${ }^{e}$ Lebedev Physical Institute, Leninsky Prospekt 53, RU-119991 Moscow, Russia.

${ }^{f}$ University of Bari, Department of Physics, Via Amendola 173, I-70126 Bari, Italy.

${ }^{g}$ INFN, Sezione di Bari, Via Amendola 173, I-70126 Bari, Italy.

${ }^{h}$ INFN, Sezione di Trieste, Padriciano 99, I-34012 Trieste, Italy.

${ }^{i}$ Ioffe Physical Technical Institute, Polytekhnicheskaya 26, RU-194021 St. Petersburg, Russia.

'University of Rome "Tor Vergata", Department of Physics, Via della Ricerca Scientifica 1,

I-00133 Rome, Italy.

${ }^{k}$ KTH, Department of Physics, and the Oskar Klein Centre for Cosmoparticle Physics, AlbaNova University Centre, 10691 Stockholm, Sweden.

'INFN, Sezione di Roma "Tor Vergata", Via della Ricerca Scientifica 1, I-00133 Rome, Italy.

${ }^{m}$ Moscow Engineering and Physics Institute, Kashirskoe Shosse 31, RU-11540 Moscow, Russia.

${ }^{n}$ IFAC, Via Madonna del Piano 10, I-50019 Sesto Fiorentino, Florence, Italy.

${ }^{\circ}$ University of Trieste, Department of Physics, Via Valerio 2, I-34127 Trieste, Italy.

${ }^{p}$ University of Siegen, D-57068 Siegen, Germany.

${ }^{q}$ INFN, Laboratori Nazionali di Frascati, Via Enrico Fermi 40, I-00044 Frascati, Italy.

In this paper we present the latest results of the Pamela satellite experiment, focusing in particular on the $\bar{p} / p$ and the $e^{+} /\left(e^{+}+e^{-}\right)$ratios.

The 2009 Europhysics Conference on High Energy Physics,

July 16 - 222009

Krakow, Poland 


\section{Introduction}

The Pamela experiment [1] has been designed as a wide range cosmic rays observatory, to allow extensive measurement of various cosmic ray species around the earth. The main physics objectives of Pamela are the precise and high statistic measurement of $\bar{p}$ and $e^{+}$spectra, in a wide energy range (from $\simeq 100 \mathrm{MeV}$ up to $\simeq 200 / 300 \mathrm{GeV}$ ). The experiment is however able to significantly contribute to increase the existing knowledge in many other cosmic ray related fields, like protons, light nuclei and electrons spectra, solar and geomagnetic physics.

Pamela has been placed in a low earth, quasi polar, orbit on June 15, 2006, on board of the Russian Resurs-DK1 satellite, launched from the Bajkonour cosmodrome (Kazakhstan). The detector performances, precisely measured on ground on various beam tests, were found to be unaffected by the launch phase; all the Pamela subystem were switched on for the physics DAQ phase few weeks after the in-orbit installation, and they are still efficiently taking data after more than three years of operations. The average trigger rate of $25 \mathrm{~Hz}$, coupled to a $73 \%$ live time, allowed more than $10^{9}$ triggers, corresponding to $14 \mathrm{~TB}$ of data downlinked to ground, to be collected.

This high statistic sample of data has given us the possibility to well understand the detector behaviour and performances, hence minimizing the systematic effects that usually limit this type of experiments.

\section{The Pamela detector}

The core of the Pamela detector (see Figure 1) is a very precise magnetic spectrometer, composed by 6 layers of silicon microstrip detectors inserted inside an uniform magnetic field volume, produced by a $140 \mathrm{~kg}$ permanent magnet. The intensity of the field $(\simeq 0.4 \mathrm{~T})$, coupled to the high precision of the silicon detector in the charged particle trajectories reconstruction $(\simeq 3 \mu \mathrm{m})$, results in a Maximum Detectable Rigidity $>1 \mathrm{TV} / \mathrm{c}$, important to increase the high energy limit in the $\bar{p}$ and $e^{+}$spectra.

Below the magnetic spectrometer a thick and highly segmented Silicon/Tungsten electromagnetic calorimeter is used to discriminate between electromagnetic and hadronic particles, by looking at the shower profile development. The very good performances of this detector in the $p$ background rejection are the key point of the $e^{+}$analysis described later on in this proceeding.

A Time Of Flight system (TOF), made by three double layers of plastic scintillator, is used both to measure the particle's velocity and to give the trigger to the experiment, while an additional anticoincidence system, surrounding the magnetic spectrometer, is used to clean up the data sample.

Two additional detector (an S4 scintillator and an ${ }^{3} \mathrm{He}$ tubes based Neutron detector), installed below the calorimeter, complete the Pamela apparatus, whose total weight amounts to $470 \mathrm{~kg}$.

\section{The $\bar{p}$ analysis}

The $\bar{p}$ analysis shown in this paper is based on 950 days statistics; the magnetic spectrometer plays the crucial role, since the most critical background in the $\bar{p}$ sample comes from spillover

\footnotetext{
* Speaker.
} 


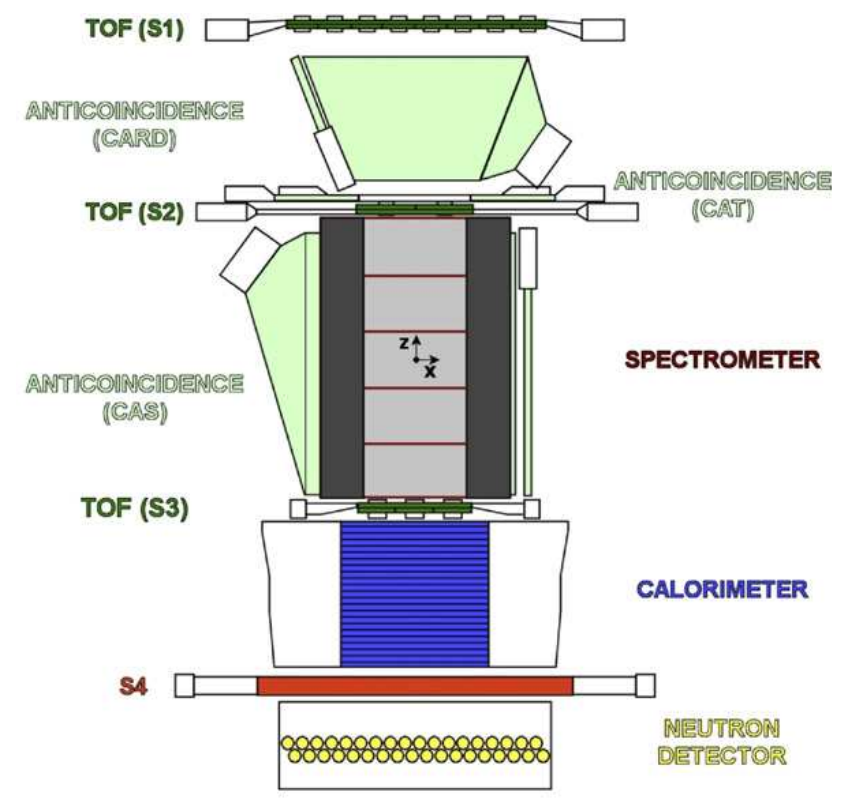

Figure 1: Schematic drawing of the Pamela apparatus.

protons (high energy protons, whose momentum is wrongly measured, hence contaminating the negative $\bar{p}$ sample).

A first and simple preselection is done by requiring clean events, without any interactions in the anticoincidence system, and with a simple hit patter in the TOF and in the magnetic spectrometer. To select galactic cosmic rays, we reject the albedo particles and we impose a rigidity measurement well above the local geomagetic cutoff. The calorimeter information is used to reject the electron background, by requiring that the energy measured in the calorimeter is not compatible with the tracker reconstructed momentum. At this point stronger selection criteria are imposed on the reconstructed track parameters, to select a very pure $\bar{p}$ sample, imposing a reconstructed momentum much smaller than the Maximum Detectable Momentum, measured on an event by event basis. The sample left in the analysis can be efficiently used to measure the $\bar{p} / p$ ratio in various energy bins. Figure 2 shows the result obtained in the $600 \mathrm{MeV} / 100 \mathrm{GeV}$ energy range; the lines indicates the theoretical expectations from the pure secondary productions models. We can clearly state that in the explored energy region the Pamela data do not show any significant deviations from the secondary production expectations.

\section{The $e^{+}$analysis}

The most critical aspect of the $e^{+}$analysis is the rejection of the huge proton background; Pamela accomplish this task mainly using the information coming from the highly segmented imaging calorimeter, that is able to identify the interacting protons in the $e^{+}$sample by looking both at the longitudinal and lateral shower development. This rejection is particularly difficult for two reasons: 


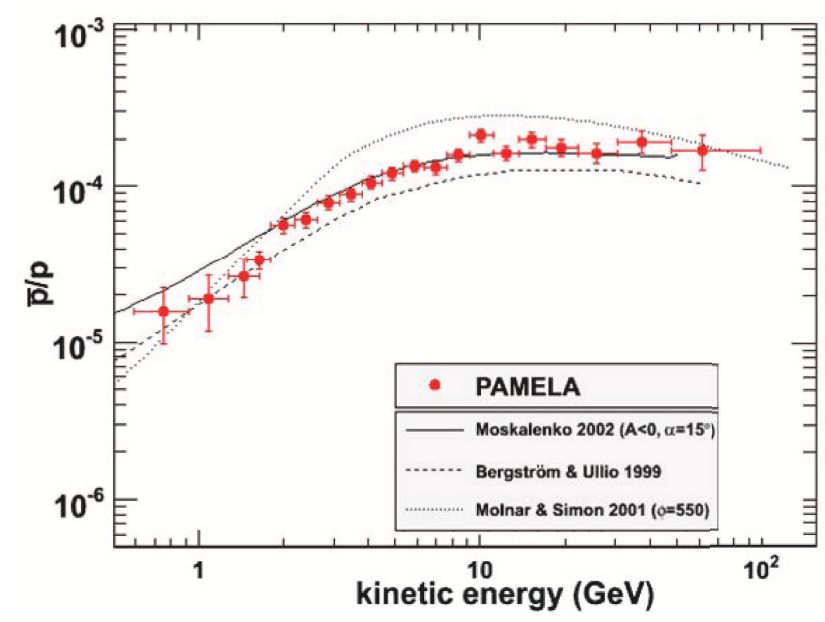

Figure 2: $\bar{p} / p$ ratio measured by Pamela with 950 days of data taking. The lines superimposed over the plot describe the expectations from the most commonly quoted pure secondary productions models.

1. the proton spectrum is harder that the $e^{+}$one, so the proton background increase with energy up to the high energy limit of the experiment $(200-300 \mathrm{GeV})$;

2. due to the big fluctuations in the hadronic shower development, it is possible that a very high energy $\pi^{0}$ can be produced in the very first layers of the calorimeter, giving raise to a dominant electromagnetic component of the shower, difficult to be distinguished from the $e^{+}$ induced showers.

A very detailed knowledge of the calorimeter is extremely important to efficiently reject and properly estimate the remaining proton contamination, and this is accomplished by means of many independent tools. First of all the calorimeter behaviour for electromagetic and hadronic particles has been studied in details before the launch at the SPS beam tests [2], clearly demonstrating a proton rejection factor greater than $10^{5}$ with an electron efficiency greater than $90 \%$. Additionaly, the Pamela simulation program has been finely tuned to well match the calorimeter information. Finally, the selected $e^{+}$sample was cross checked with some additional Pamela subdetector's information, like the tracker $\mathrm{dE} / \mathrm{dx}$ or the neutron yield measured by the neutron detector.

However, the final $e^{+}$analysis does not make any use of test beam data or simulation results, since the estimation of the proton background is directly determined by the data themselves with the pre-sampler method [3]. The calorimeter is divided in two parts, the first one $1.5 \mathrm{X}_{0}$ thick, the second one $15 \mathrm{X}_{0}$ thick. A clean control sample of protons can be identified by looking at the particles not interacting in the first calorimeter part; a clean control sample of electrons can be selected by looking at negative rigidity particles interacting in the first calorimeter part. The topological variables used for the $e^{+}$selection (like for example the fraction of energy in a \pm 0.6 Moliere Radius region around the calorimeter's track) can be computed for the two different control samples; in particular, for the proton control samples the topological variables are computed by using only the informations form the deeper $15 \mathrm{X}_{0}$ thick part, to correctly simulating the proton background. At this point, the signal and background fractions in the selected positive rigidity electromagnetic 
particles can be directly estimated from the data, by properly weighting the control samples distributions with a statistical method [4], in different rigidity bins. The resulting $e^{+} /\left(e^{+}+e^{-}\right)$ratio obtained with data gathered in 500 days is plotted in figure 3; red dots are the Pamela measured data, compared with previous experiments data and with the most commonly used calculation for pure secondary production models.

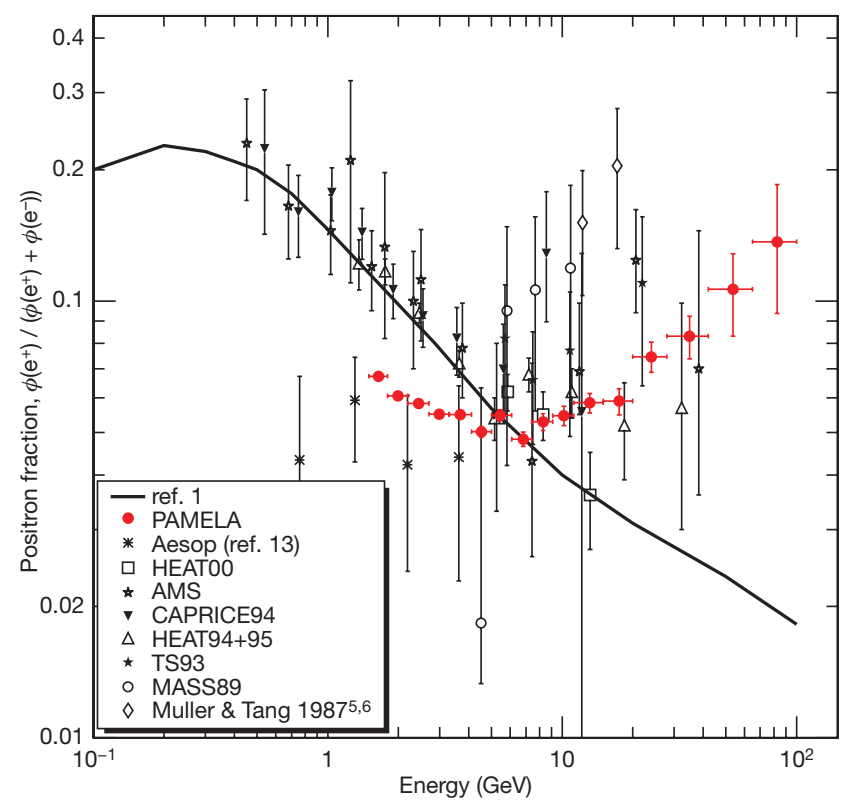

Figure 3: $e^{+} /\left(e^{+}+e^{-}\right)$ratio measured by Pamela with 500 days of data taking. The lines superimposed over the plot describe the expectations from the most commonly quoted pure secondary productions models, and are clearly incompatible with the Pamela results.

The Pamela data are clearly incompatible with the secondary production expectations; this significant discrepancy stimulated an extremely active discussion in the cosmic ray and cosmology communities. Clearly any reasonable explanation should take into account also the $\bar{p}$ results, that do not show any significant discrepancy with respect to the secondary production expectations. Generally speaking, the Pamela data could be interpreted as due to some primary positron components, related to the dark matter component of the universe, or to some not well known or modelled astrophysical sources, like for example nearby pulsars. For a review of the possible interpretations, see for example [3]

\section{Conclusions}

In this paper we have presented the results obtained with the Pamela experiment on the $\bar{p} / p$ and $e^{+} /\left(e^{+}+e^{-}\right)$ratios, in a wide energy range $(600 \mathrm{MeV}-100 \mathrm{GeV}$ for the $\bar{p}$ and $1.5 \mathrm{GeV}-100 \mathrm{GeV}$ for the $\left.e^{+}\right)$. These data shows a substantial agreement with the pure secondary model production expectations for the $\bar{p}$, while the $e^{+}$data show a clear overabundance with respect to the secondary production expectations. An active debate is actually underway in the scientific community to understand and explain these Pamela results. 
In addition to these main physics items, Pamela data allow also detailed study of many additional physics items, that span from cosmic ray propagation models (proton and Helium spectra, secondary/primary ratios etc.), to solar physics (transient solar related phenomena and solar modulation related low energy spectra), to earth and geomagnetic physics (sub-geomagnetic cutoff cosmic rays, albedo and reentrant partices). These results will soon be published in dedicated papers.

\section{References}

[1] Picozza P. et al., Astropart. Phys. 27, (2007) 296.

[2] M. Boezio et al., Astropart. Phys. 26 (2006) 111.

[3] M. Boezio et al., PAMELA and Indirect Dark Matter Searches, accepted for publication in 'New Journal of Physics'.

[4] O. Adriani et al., Nature 458, (2009) 607. 